\title{
¿Es el inventario YLS/CMI capaz de predecir la reincidencia delictiva de los menores infractores de diferentes etnias?
}

\author{
Aitana Gomis-Pomares \\ apomares@uji.es \\ Lidón Villanueva \\ bvillanu@uji.es
}

\section{Resumen}

La evaluación del riesgo en los menores infractores juveniles es crítica para reducir la posibilidad de reincidencia delictiva en un futuro. No obstante, poca literatura ha analizado la aplicabilidad de las herramientas de evaluación de riesgos para poblaciones pertenecientes a diferentes etnias. Por ello, este estudio se realizó para evaluar la validez predictiva del Youth Level of Service / Case Management Inventory (YLS/CMI) en su versión española (IGI), en dos grupos étnicos diferentes en España. Para abordar este tema, se completó el inventario para una muestra de menores infractores de etnia árabe $(N=116)$ y menores infractores de etnia gitana $(N=91)$. Los resultados de estos grupos se compararon con los obtenidos por los menores infractores que no pertenecían a ninguna de estas etnias $(\mathrm{N}=140)$. Todos los participantes tenían edades comprendidas entre 14 y 17 años. Para evaluar la reincidencia delictiva, se analizaron los expedientes posteriores después de la fecha de la primera evaluación llevada a cabo por el Equipo Técnico de Menores de Castellón (período de seguimiento: del 2012 al 2017). En primer lugar, se realizó una serie de pruebas t para muestras independientes para examinar posibles variaciones en la puntuación total del YLS/CMI para los diferentes grupos de menores infractores. Además, se realizó la curva ROC y el análisis de regresión logística para determinar la validez predictiva del inventario YLS/CMI. Aunque el inventario parece ser un instrumento de riesgo capaz de predecir la reincidencia entre todos los grupos, apunta a una evaluación de riesgos más precisa para el grupo no étnico en comparación con el resto de los grupos. Esto puede indicar que la variable étnica es un factor que los profesionales deben tener en cuenta a la hora de usar este tipo de evaluaciones de riesgo.

Palabras clave: etnia, menores infractores, validez predictiva, YLS/CMI, reincidencia. 


\begin{abstract}
The evaluation of risk in juvenile offenders is critical to reduce the possibility of criminal recidivism in the future. However, little literature has analyzed the applicability of risk assessment tools for populations belonging to different ethnic groups. Therefore, this study was conducted to evaluate the predictive validity of the Youth Level of Service / Case Management Inventory (YLS/CMI) in its Spanish version (IGI), in two different ethnic groups in Spain. To address this issue, the Inventory was completed for a sample of young Arab offenders $(\mathrm{N}=116)$ and young offenders of the Roma ethnic group $(\mathrm{N}=91)$. The results of these groups were compared with those obtained by the minor offenders who did not belong to any of these ethnic groups $(N=140)$. All the participants were between the ages of 14 and 17 years old. To evaluate the criminal recidivism, the subsequent files were analyzed after the date of the first evaluation carried out by the Castellón Minors Technical Team (follow-up period: 2012 to 2017). First, a series of $t$ tests for independent samples was conducted to examine possible variations in the total YLS/CMI score for different groups of juvenile offenders. In addition, the ROC curve and the logistic regression analysis were performed to determine the predictive validity of the YLS/CMI inventory. Although the inventory seems to be a risk instrument capable of predicting recidivism among all groups, it points to a more accurate risk assessment for the non-ethnic group compared to the rest of the groups. This may indicate that the ethnic variable is a factor that professionals must take into account when using this type of risk assessment.
\end{abstract}

Keywords: ethnicity, minor offenders, predictive validity, YLS/CMI, recidivism.

\title{
Introducción
}

Muchos investigadores han señalado la gran importancia que tiene la evaluación del riesgo de los menores infractores para reducir la reincidencia delictiva futura y establecer objetivos y tratamientos adecuados en cada caso (Holsinger, Lowenkamp y Latessa 2003). Hasta el momento, se han llevado a cabo varios estudios para evaluar la validez de diferentes instrumentos de evaluación de riesgos para la reincidencia con el objetivo de garantizar una evaluación y un uso adecuados de tales instrumentos (Olver, Stockdale y Wong 2012; Wormith, Hogg y Guzzo 2015).

Inicialmente, la evaluación de la predicción del comportamiento delictivo se llevaba a cabo a través del juicio clínico. Los motivos de las decisiones eran subjetivos, a veces intuitivos y no validados empíricamente (Cuervo et al. 2009). Luego, se elaboraron escalas de riesgo estático, demostrándose que estas escalas son más exhaustivas en comparación con el juicio clínico (Andrews, Bonta y Wormith 2006). No obstante, las escalas estáticas no se basan en una teoría y no se tienen en cuenta los aspectos dinámicos de los menores, que son los únicos que pueden ser modificables con intervención. En consecuencia, aparece un período en el que la evaluación de riesgos se llevó a cabo por escalas de riesgo/necesidad. Con estas escalas, los motivos de la decisión se medían de forma sistemática y objetiva (Andrews, Bonta y Wormith 2006; Shepherd y Sullivan 2017). Finalmente, las evaluaciones de riesgo de los menores comenzaron a enfatizar la necesidad de relacionar la evaluación y la intervención, maximizando los beneficios del tratamiento (Cuervo y Villanueva 2013). Uno de los métodos utilizados actualmente para determinar la evaluación de riesgos que se incluye en esta perspectiva es el inventario Youth Level Service / Case Management 
Inventory (YLS/CMI), en su versión para jóvenes, que procede del inventario Level Service Inventory-Revised (LSI-R) de Andrews y Bonta (1995).

El inventario de YLS/CMI se basa en el modelo general psicológico, social y de personalidad de la conducta delictiva (Andrews, Bonta y Wormith 2006). Este modelo indica que, de acuerdo con numerosos estudios sobre la predicción de la reincidencia delictiva (Bonta, Law y Hanson 1998; Gendreau, Little y Goggin 1996), los factores con mayor valor predictivo serían las actitudes antisociales, las amistades antisociales, la personalidad antisocial y los delitos previos, que constituyen los llamados big four (Andrews, Bonta y Wormith 2006). Estos estudios citados anteriormente, junto con muchos otros (Lipsey y Derzon 1998; Loeber y Stouthamer-Loeber 1987), también indican que estos cuatro factores irían seguidos por otro grupo de factores con correlaciones moderadas, que son las circunstancias familiares deficientes, la educación y el empleo, el abuso de sustancias y el ocio y el tiempo libre. Juntos, todos estos factores se conocen como los central eight (Andrews y Bonta 2010). Con respecto a la validez del inventario $\mathrm{YLS} / \mathrm{CMI}$, algunos estudios han demostrado que este instrumento predice la reincidencia delictiva de manera adecuada, convirtiéndose en una de las mejores opciones para evaluar el riesgo de reincidencia en menores (Olver, Stockdale y Wormith 2009; Cuervo y Villanueva 2015; Gendreau, Little y Goggin 1996).

De modo que, a pesar del auge en el uso de los instrumentos de evaluación de riesgos en los últimos años, la cantidad de investigación con minorías étnicas sigue siendo relativamente escasa. La cultura de una persona juega un papel importante en la manifestación del riesgo. La cultura determina las normas de comportamiento y las expectativas así como la delincuencia (Shepherd y Lewis-Fernandez 2016). Los instrumentos de evaluación de riesgos a menudo se elaboran dentro de los preceptos de un grupo cultural, ignorando la amplia variabilidad experiencial en diferentes etnias, y las estimaciones de predicción a menudo son menos precisas para los grupos minoritarios étnicos (Holsinger, Lowenkamp y Latessa 2003; Thompson y McGrath 2012; Wormith, Hogg y Guzzo 2015). Esto resulta poco ventajoso para algunos grupos minoritarios étnicos que se encuentran sobrerrepresentados en el sistema judicial.

La falta de investigación sobre la utilidad del inventario YLS/CMI en las minorías étnicas es una omisión importante por varias razones. Si el rango de marcadores de riesgo disponibles como objetivos de tratamiento no se ha probado adecuadamente en una población determinada, esta información puede ser poco informativa o inexacta al desarrollar estrategias de reducción de riesgos y gestión de riesgos para esa población (Shepherd, Singh y Fullam 2015).

Algunos autores (Bonta 1989; Wilson y Gutierrez 2014; Wormith, Hogg y Guzzo 2015) han realizado estudios para examinar los instrumentos de evaluación de riesgos en las minorías étnicas adultas. Por ejemplo, en 1989, Bonta examinó la validez predictiva de la LSI con reclusos aborígenes y no aborígenes y encontró que, a pesar de que la predictibilidad general de la reincidencia no mostraba grandes diferencias entre estos dos grupos, había algunas diferencias en aspectos financieros o en las necesidades de alojamiento, que actuaban mejor como variables predictoras para los delincuentes no aborígenes. En la misma línea, Wilson y Gutierrez (2014) realizaron un metaanálisis para examinar la validez predictiva de las subescalas del instrumento LSI en muestras de delincuentes aborígenes y no aborígenes. Los resultados mostraron que algunos factores (antecedentes penales, abuso de sustancias y patrón de personalidad antisocial) se asociaron significativamente con la reincidencia general para los delincuentes aborígenes, pero no de forma tan intensa como lo fueron para los delincuentes no aborígenes. Estudios como el llevado a cabo por Holsinger, Lowenkamp y Latessa (2003) enfatizan las diferencias existentes en la predicción de 
LSI-R en función de la etnia y el género. En este sentido, un estudio reciente de Wormith, Hogg y Guzzo (2015), que analiza la utilidad de un instrumento general de riesgo/necesidad (LS) con delincuentes aborígenes en Canadá, muestra una mejor predicción de reincidencia para los delincuentes no aborígenes que los delincuentes aborígenes. Al mismo tiempo, el estudio reveló que los delincuentes aborígenes presentaban un mayor nivel de factores de riesgo y generalmente reincidían más que el grupo no aborigen.

A pesar de que se han analizado diferentes instrumentos de predicción de reincidencia delictiva en adultos, hasta el momento muy pocos estudios han validado los instrumentos de predicción de reincidencia delictiva en menores infractores pertenecientes a grupos étnicos (Rembert, Henderson y Pirtle 2014).

Por un lado, si analizamos las minorías étnicas mayoritarias en España, encontramos que una de las minorías étnicas más habituales es la población gitana, que representa el $21,3 \%$ de los menores infractores (Uceda-Maza y Domínguez Alonso 2017). De hecho, según el Ministerio de Sanidad, Servicios Sociales e Igualdad de España (2010), la población gitana que se estima que reside en España es de alrededor de 700000 y 970000 personas, lo que representa aproximadamente el $1,5 \%$ del total de la población española (La Parra, Gil-González y Jiménez 2013). Al mismo tiempo, la población gitana que vive en la Comunidad Valenciana sería de alrededor de 55000 habitantes, siendo el rango de edad mayoritaria entre 15 y 19 años (CIS 2007).

Por otro lado, otra de las minorías étnicas más destacadas en España es la población árabe (INE 2017). Cuando se habla de menores, los menores infractores árabes representan el 6-11\% de la población total de jóvenes delincuentes en España y se ha observado que duplican la tasa de reincidencia española (40\% frente a $20 \%$ ) (Capdevila, Ferrer y Luque 2005; Cuervo, Villanueva y Prado-Gascó 2017). Un estudio realizado en Cataluña (España) incluso defiende que los menores infractores del Magreb son el grupo con más factores de riesgo y menos factores de protección, es decir, el grupo con el perfil criminal y criminológico más difícil (Capdevila, Ferrer y Luque 2005). Por esta razón, los instrumentos de evaluación de riesgos deben ser capaces de demostrar su utilidad en todos los grupos culturales para evitar clasificaciones erróneas y reflejar realmente la población que se está midiendo. Sin embargo, pocos estudios han analizado la validez predictiva de los instrumentos de evaluación de riesgos en minorías étnicas.

Por lo tanto, el objetivo de este estudio es explorar la aplicabilidad y la validez del YLS/CMI en dos minorías étnicas: la minoría árabe y la minoría gitana. Se hipotetiza que la validez predictiva del YLS/CMI será más precisa para el grupo no minoritario que para los dos grupos minoritarios. En segundo lugar, se espera que los menores infractores de procedencia árabe y gitana presenten tasas más elevadas de reincidencia delictiva, más factores de riesgo y menos factores de protección en comparación con el grupo no étnico.

\section{Método}

\section{Participantes}

La muestra total consistió en 347 menores infractores, de entre 14 y 17 años (media $=15,84$ años, DT $=1063$ ), de la provincia de Castellón, en España. Todos ellos habían cometido un delito u ofensa y, por lo tanto, fueron entrevistados por el Equipo Técnico de Menores durante el período comprendido entre 2012 y 2017 . De la muestra total, 88 eran mujeres y 259 eran hombres, representando el $25,4 \%$ y el $74,6 \%$, 
respectivamente. Los participantes fueron seleccionados en función de su origen étnico. El criterio escogido fue la autoidentificación de los menores como gitanos, como árabes o como no pertenecientes a ninguna minoría étnica.

En primer lugar, el grupo gitano estaba formado por 91 sujetos, 29 de los cuales eran mujeres y 62 hombres $(31,9 \%$ y $68,1 \%)$. La edad promedio fue de 15,88 años (DT $=1,09)$. En segundo lugar, el grupo árabe consistió en 116 sujetos, de los cuales 14 eran mujeres y 102 hombres $(12,1 \%$ y $87,9 \%)$. La edad media fue de 15,76 años $(D T=1,09)$. En tercer lugar, el grupo no étnico estaba constituido por 140 participantes, de los cuales 45 eran mujeres y 95 hombres $(32,1 \%$ y $67,9 \%)$, con una edad promedio de 15,88 años (DE $=1,01)$.

\section{Instrumento}

El instrumento que se utilizó en el estudio fue el Youth Level Service / Case Management Inventory (YLS/CMI) de Hoge y Andrews (2002), que fue traducido por Garrido, López, Silva, López y Molina (2006) como el «Inventario de gestión e intervención para jóvenes» (IGI-J). El objetivo del inventario es evaluar el riesgo de reincidencia juvenil basada en la información recopilada de diferentes fuentes.

El inventario consta de 42 ítems, que se pueden clasificar de acuerdo a 8 factores de riesgo. En cada factor, el evaluador señala el número de ítems que encuentra presentes en el menor en cada área: 1) delitos y medidas judiciales pasadas y actuales (incumplimientos y quebrantamientos de medidas judiciales); 2) pautas educativas (disciplina parental inapropiada, pautas educativas inconsistentes); 3) educación formal y empleo (comportamiento disruptivo en clase, bajo rendimiento académico); 4) grupo de iguales antisociales (algunos de sus conocidos son delincuentes, pocos conocidos son modelos positivos); 5) consumo de sustancias (consumo habitual de drogas o alcohol); 6) ocio/diversión (pocas actividades organizadas, malgasta su tiempo de ocio); 7) personalidad/conducta (agresividad física, sentimientos de culpa inadecuados); 8) actitudes, valores, creencias (actitudes antisociales, desafía a la autoridad). A partir de la suma de todos los ítems presentes en el menor, se obtiene un nivel de riesgo de reincidencia, siendo las franjas de riesgo en las que se puede clasificar a los menores las siguientes: bajo (0-8 puntos), moderado (9-22), alto (23-32) y muy alto (33-42).

La versión española del inventario ha mostrado propiedades psicométricas adecuadas en estudios previos, obteniendo un alfa de Cronbach que oscila de 0,87 (Cuervo y Villanueva 2013) a 0,91 (Cuervo, Villanueva y Prado-Gascó 2017) para todos los ítems del inventario. En este estudio, el alfa de Cronbach fue 0,85.

\section{Procedimiento}

Los datos para este estudio se obtuvieron del análisis de los registros del Juzgado de Menores de la provincia de Castellón. El análisis incluyó el número de delitos de cada menor en un período de seguimiento que abarcó desde el 2012 hasta el 2017. Se recopilaron datos demográficos relacionados con la etnia, la nacionalidad y el género y el riesgo de reincidencia juvenil que se obtuvo mediante el inventario YLS/CMI. Primero, se obtuvo la muestra de menores infractores de etnia gitana (91 sujetos) y posteriormente la árabe (116 sujetos). El grupo de comparación que no pertenecía a ninguna minoría étnica se seleccionó mediante un procedimiento aleatorio hasta obtener una muestra total formada por 347 sujetos. 
La variable reincidencia delictiva hace referencia a los expedientes posteriores a la fecha de la primera evaluación llevada a cabo en el menor por el Equipo Técnico de Menores. Esta variable se codificó en formato binario $(0=\sin$ reincidencia, $1=$ reincidencia).

\section{Análisis de datos}

En primer lugar, se realizó la prueba ANOVA para examinar la existencia de diferencias significativas en las puntuaciones totales del YLS/CMI tanto para los factores de riesgo como para los factores protectores para cada uno de los grupos. Por otra parte, el análisis de fiabilidad se centró en la evaluación de la consistencia interna utilizando alfa de Cronbach (1951). Se analizó la curva ROC y la regresión logística binaria para determinar la validez predictiva del inventario.

\section{Resultados}

Los resultados de los factores de riesgo en las diferentes áreas del inventario indicaron que los grupos muestran diferencias significativas en todas las áreas del inventario excepto en el área de consumo de sustancias y personalidad/conducta. Se observa que, generalmente, las medias son más altas en el grupo gitano y más bajas en el grupo árabe. Con respecto a los factores protectores, se observaron diferencias significativas en las áreas de grupo de iguales antisociales, consumo de sustancias y puntuación total en factores protectores. Las medias más altas las obtienen el grupo árabe y el grupo no étnico, indicando un mayor nivel de factores protectores en comparación con el grupo gitano.

Por otro lado, los análisis de consistencia interna (alfa de Cronbach) mostraron una confiabilidad de 0,848 para la muestra árabe, de 0,76 para el grupo gitano y de 0,855 para el grupo no étnico.

También se realizó un análisis del área bajo la curva (AUC) para evaluar la capacidad de la puntuación total de ocho factores del Instrumento. Un valor de AUC de 0,50 indica una predicción debida al azar y un valor de 1 indica una predicción perfecta. En este caso, se observó un AUC de 0,73 (DT =0,05) para el grupo árabe, un AUC de $0,69(\mathrm{DT}=0,06)$ para el grupo gitano y un AUC de 0,76 $(\mathrm{DT}=0,04)$ para el grupo no étnico.

A continuación, se presentan los resultados de la regresión logística binaria para el grupo gitano (tabla 1). Como se puede observar, el modelo final fue estadísticamente significativo y explicó el $21,7 \%$ de la varianza en la predicción de la reincidencia. Ni la puntuación total en los factores protectores ni la edad o el sexo fueron significativos para el modelo total. 
Tabla 1

Análisis de regresión logística de la reincidencia para menores delincuentes gitanos MODELO 3: FACTORES DE RIESGO Y FACTORES PROTECTORES

$95 \% \mathrm{Cl}(\mathrm{B})$

\begin{tabular}{ccccccccc} 
& $\mathrm{B}$ & $\mathrm{SE}$ & Wald & $\mathrm{df}$ & $\mathrm{p}$ & $\mathrm{Exp}(\mathrm{B})$ & $\mathrm{LL}$ & $\mathrm{UL}$ \\
\hline Mujer(1) & $-0,85$ & 0,56 & 2,31 & 1 & 0,128 & 0,426 & 0,14 & 1,29 \\
Edad & 0,09 & 0,23 & 0,17 & 1 & 0,680 & 1,09 & 0,71 & 1,70 \\
Riesgo total & 0,15 & 0,05 & 7,27 & 1 & $0,007^{*}$ & 1,16 & 1,04 & 1,29 \\
Factores protectores & 0,51 & 0,85 & 0,36 & 1 & 0,549 & 1,66 & 0,31 & 8,77 \\
Constante & $-3,77$ & 3,79 & 0,98 & 1 & 0,321 & 0,023 & & \\
\hline
\end{tabular}

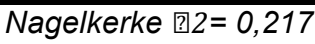

En segundo lugar, para el grupo de árabes (tabla 2), en la regresión indicó que la puntuación de riesgo total fue la variable que más contribuyó al modelo final. El modelo final fue estadísticamente significativo y explicó el $32,6 \%$ de la varianza en la predicción de la reincidencia. A su vez, el género también fue una variable significativa para el modelo total.

Tabla 2

Análisis de regresión logística de la reincidencia para menores delincuentes árabes

MODELO 3: FACTORES DE RIESGO Y FACTORES PROTECTORES

$95 \% \mathrm{Cl}(\mathrm{B})$

\begin{tabular}{ccccccccc} 
& $\mathrm{B}$ & $\mathrm{SE}$ & Wald & $\mathrm{df}$ & $\mathrm{p}$ & $\mathrm{Exp}(\mathrm{B})$ & $\mathrm{LL}$ & $\mathrm{UL}$ \\
\hline Mujer(1) & $-0,63$ & 0,85 & 0,55 & 1 & 0,457 & 0,532 & 0,10 & 2,81 \\
Edad & $-0,28$ & 0,22 & 1,62 & 1 & 0,203 & 0,755 & 0,49 & 1,16 \\
Factores de riesgo & 0,15 & 0,47 & 11,07 & 1 & $0,001^{*}$ & 1,17 & 1,07 & 1,28 \\
Factores protectores & 0,30 & 0,33 & 0,81 & 1 & 0,369 & 1,35 & 0,69 & 2,62 \\
Constante & 2,04 & 3,49 & 0,34 & 1 & 0,559 & 7,72 & & \\
\hline
\end{tabular}

Nagelkerke $R^{2}=0,211$

Por otro lado, los análisis de regresión obtenidos para la muestra de menores infractores del grupo no étnico se muestran en la tabla 3. Al igual que en los casos anteriores, la puntuación de riesgo total fue la variable que contribuyó significativamente al modelo final. Además, el género también fue una variable significativa. El modelo final explica el $32,6 \%$ de la varianza en la predicción de la reincidencia. Por otro lado, la puntuación total en los factores protectores no fue una variable significativa en este modelo. 
Tabla 3

Análisis de regresión logística de la reincidencia para menores delincuentes del grupo no étnico.

MODELO 3: FACTORES DE RIESGO Y PROTECTORES

\begin{tabular}{ccccccccc} 
& & & & & & & \multicolumn{3}{c}{$95 \%$ Cl (B) } \\
& B & SE & Wald & df & p & Exp (B) & LL & UL \\
\hline Mujer(1) & $-1,21$ & 0,55 & 4,81 & 1 & $0,028^{*}$ & 0,299 & 0,10 & 1,02 \\
Edad & $-0,06$ & 0,22 & 0,07 & 1 & 0,785 & 0,943 & 0,62 & 1,44 \\
Factores de riesgo & 0,16 & 0,04 & 17,53 & 1 & $0,000^{*}$ & 1,17 & 1,08 & 0,984 \\
Factores protectores & $-0,15$ & 0,35 & 0,18 & 1 & 0,668 & 0,859 & 0,43 & 1,72 \\
Constante & $-1,26$ & 3,13 & 0,00 & 1 & 0,715 & 0,951 & & \\
\hline
\end{tabular}

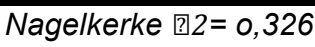

\section{Discusión y conclusiones}

El presente estudio examinó el uso del inventario YLS/CMI en dos muestras de menores infractores pertenecientes a diferentes grupos étnicos (gitanos y árabes). En primer lugar, se formuló la hipótesis de que la validez predictiva del YLS/CMI sería más precisa para el grupo no étnico que para los grupos étnicos (gitano y árabe). Esta hipótesis no fue totalmente respaldada por los resultados. Con diferencias muy sutiles, el inventario mostró una validez predictiva similar tanto para el grupo no étnico como para el grupo árabe. Sin embargo, la validez predictiva para el grupo gitano fue inferior que para el resto de grupos.

Los valores de los análisis ROC y los modelos de regresión para el inventario YLS/CMI siempre fueron ligeramente más altos en el grupo no étnico en comparación con el grupo árabe y gitano (AUC $=0,76 ; 0,73 ; 0,69 ; R^{2}=0,33 ; 0,21 ; 0,21$ respectivamente). Para todos los grupos, los valores de AUC obtenidos en este estudio se encontraban en el rango superior en comparación con estudios previos (Shepherd, Singh y Fullam 2015).

En los diferentes modelos de regresión, la variable factores de riesgo fue consistentemente un predictor significativo de reincidencia en todos los grupos. Para todos los participantes en este estudio, e independientemente de la cultura, la puntuación total en los factores de riesgo en el inventario YLS/CMI fue capaz de predecir la reincidencia posterior durante el período de seguimiento.

En segundo lugar, se esperaba que los menores infractores pertenecientes a los grupos étnicos presentasen más factores de riesgo y menos factores de protección que el grupo no étnico. En el caso del grupo gitano, esta hipótesis se cumplió; sin embargo, los menores infractores árabes no presentaron más factores de riesgo ni menos factores de protección que los menores infractores del grupo no étnico. Así pues, esta investigación muestra que el valor total obtenido del inventario de YLS/CMI es un predictor adecuado de la reincidencia general en todas las muestras. Sin embargo, el YLS/CMI presenta una validez predictiva más precisa para el grupo no étnico que para los grupos étnicos. Por tanto, el presente estudio realizado con dos grupos minoritarios españoles (población gitana y árabe) apoya los resultados obtenidos por estudios previos con aborígenes, afroamericanos o minorías del Pacífico, mostrando consistentemente una validez predictiva más débil del inventario 
YLS/CMI en estos grupos (Lidell, Blake y Singh 2016; Onifade, Davidson y Campbell 2009; Schwalbe, Fraser, Day y Cooley 2006; Wilson y Gutierrez 2014; Wormith, Hogg y Guzzo 2015).

\section{Conclusiones}

En conclusión, de acuerdo con los resultados obtenidos, el inventario YLS/CMI ha demostrado evaluar correctamente la reincidencia en menores infractores gitanos, árabes y menores no pertenecientes a ningún grupo étnico. Sin embargo, debe tenerse en cuenta que la evaluación no es tan precisa en todos los casos. Por ello, estos resultados presentan implicaciones importantes para los profesionales que llevan a cabo la evaluación del riesgo de menores ya que, si no se realiza una evaluación precisa en una población en particular, pueden darse errores de precisión o clasificación. Por lo tanto, los profesionales deben ser conscientes de las diferencias culturales y deben traducir esa conciencia en la prestación de servicios que sean culturalmente sensibles. La validación de instrumentos de riesgo interculturales y la literatura de factores de riesgo culturalmente específicos aún están en desarrollo, por lo que es importante que las personas que trabajan con estos grupos logren esta competencia cultural (Shepherd, Singh y Fullam 2015).

\section{Referencias bibliográficas}

Andrews, David A. y James Bonta. 1995. LSI-R: The Level of Service InventoryRevised. Toronto: Multi-Health Systems. http://doi.org/10.1177/0011128705281756

- 2010. The psychology of criminal conduct (5th ed.). New Providence, NJ: LexisNexis

Andrews, David A., James Bonta y J. Stephen Wormith. 2006. «The recent past and near future of risk and/or need assessment». Crime and Delinquency 52: 7-27.

Bonta, James. 1989. "Native inmates: Institutional response, risk, and needs». Canadian Journal of Criminology 31: 49-62.

Bonta, James, Moira Law y Karl Hanson. 1998. «The prediction of criminal and violent recidivism among mentally disordered offenders: A meta-analysis». Psychological Bulletin 123(2): 123-142.

Capdevila, Manel, Marta Ferrer y Eulàlia Luque. 2005. La reincidencia en el delito en la justicia de menores. Centre d'estudis Jurídics i formació especialitzada. Barcelona: Generalitat de Catalunya.

CIS. 2007. Encuesta Sociológica a Hogares de la Población Gitana. Madrid: ESTUDIO CIS.

Cronbach, Lee J. 1951. "Coefficient alpha and the internal structure of tests». Psychometrika 16: 297-334. http://doi.org/10.1007/BF02310555

Cuervo, Keren, Clara Andrés, Ana Belén Górriz, María Lidón Villanueva, Cruz Carrión y María del Pilar Busquets. 2009. "Predicción de la reincidencia delictiva en menores infractores». International Journal of Developmental and Educational Psychology 1: 529-537.

Cuervo, Keren y María Lidón Villanueva. 2013. «Reiteración y reincidencia delictivas en menores españoles con expediente Judicial». Revista Mexicana de Psicología 30: 61-68.

- 2015. "Analysis of risk and protective factors for recidivism in Spanish youth offenders». International Journal of Offender Therapy and Comparative Criminology 59: 1149-1165. http://doi.org/10.1177/0306624X14557917 
Cuervo, Karen, María Lidón Villanueva y Vicente Javier Prado-Gascó. 2017. «Predicción de la reincidencia juvenil mediante el inventario YLS/CMI y baremos para su valoración». Revista Mexicana de Psicología 34: 24-36.

Garrido, Vicente, Enrique López, Teresa Silva, M. ${ }^{a}$ Jesús López y Pedro Molina. 2006. El modelo de la competencia social de la ley de menores. Valencia: Tirant Lo Blanc.

Gendreau, Paul, Tracy Little y Claire Goggin. 1996. «A meta-analysis of the predictors of adult offender recidivism: What works!». Criminology 34: 575-607

Holsinger, Alexander M., Christopher T. Lowenkamp y Edward J. Latessa. 2003. «Ethnicity, gender, and the Level Service Inventory-Revised». Journal of Criminal Justice 31: 309-320. http://doi.org/10.1016/S0047-2352(03)00025-4

Informe del Instituto Nacional de Estadística. 2017. Estadística de migraciones 2016. Madrid: Instituto Nacional de Estadística.

La Parra, Daniel, Diana Gil-González y Antonio Jiménez. 2013. "Los procesos de exclusión social y la salud del pueblo gitano en España». Gaceta Sanitaria 27(5): 385-386. http://doi.org/10.1016/j.gaceta.2013.05.001

Lidell, Marg, Meredith Blake y Supriya Singh. 2016. "Over-represented and misunderstood: Pacific young people and juvenile justice in NSW». Australian \& New Zealand Journal of Criminology 50(4): 1-19. http://doi.org/10.1177/0004865816666614

Lipsey, Mark W. y James H. Derzon. 1998. «Predictors of violent or serious delinquency in adolescence and early adulthood: A synthesis of longitudinal research». En Serious and violent juvenile offenders: Risk factors and successful interventions, ed. Rolf Lober y David P. Farrington (86-105). Thousand Oaks, CA: Sage Publications.

Loeber, Rolf y Magda Stouthamer-Loeber. 1987. «Prediction». En Handbook of juvenile delinquency, ed. Herbert C. Quay (313-345). New York: John Wiley and Sons.

Ministerio de Sanidad, Servicios Sociales e Igualdad. 2010. Diagnóstico social de la comunidad gitana en España. Un análisis contrastado de la Encuesta del CIS a Hogares de Población Gitana 2007. Madrid: Cyan, Proyectos Editoriales.

Olver, Mark E., Keira C. Stockdale y Stephen C. P. Wong. 2012. "Short and long-term prediction of recidivism using the Youth Level of Service/Case Management Inventory in a sample of serious young offenders». Law and Human Behavior 36: 331-344. http://doi.org/10.1037/h0093927

Olver, Mark E., Keira C. Stockdale y J. Stephen Wormith. 2009. «Risk assessment with young offenders: A meta-analysis of three assessment measures». Criminal Justice and Behavior 36: 329-353. http://doi.org/10.1177/0093854809331457

Onifade, Eyitayo, William Davidson y Christina Campbell. 2009. «Risk assessment: The predictive validity of the youth level of service case management inventory with African Americans and girls». Journal of Ethnicity in Criminal Justice 7(3): 205-221. http://doi.org/10.1080/15377930903143544

Rembert, David A., Howard Henderson y Danny Pirtle. 2014. «Differential racial/ethnic predictive validity». Youth Violence and Juvenile Justice 12: 152-166. doi: 10.1177/1541204013485606

Schwalbe, Craig S., Mark W. Fraser, Steven H. Day y Valerie Cooley. 2006. "Classifying juvenile offenders according to risk of recidivism: Predictive validity, race/ethnicity, and gender». Criminal Justice and Behavior 33: 305-324.

Shepherd, Stephane M. y Roberto Lewis-Fernandez. 2016. «Forensic risk assessment and cultural diversity: Contemporary challenges and future directions». Psychology, Public Policy, and Law 22: 427. http://doi.org/10.1037/law0000102

Shepherd, Stephane M., Jay P. Singh y Rachael Fullam. 2015. «Does the Youth Level of Service/Case Management Inventory generalize across ethnicity?». International Journal of Forensic Mental Health 14: 193-204. http://doi.org/10.1080/14999013.2015.1086450 
Shepherd, Stephane M. y Danny Sullivan. 2017. «Covert and implicit influences on the interpretation of violence risk instruments». Psychiatry, Psychology and Law 24: 292-301.

Thompson, Anthony P. y Andrew McGrath. 2012. "Subgroup differences and implications for contemporary risk-need assessment with juvenile offenders". Law and Human Behavior 36: 345-355. http://doi.org/10.1037/ h0093930

Uceda-Maza, Francesc Xavier y Francisco Javier Domínguez Alonso. 2017. «Vinculación entre la vulnerabilidad y la exclusión social y las trayectorias delictivas. Un estudio de asociación». Psychosocial Intervention 26: 29-37. http://doi.org/10.1016/j.psi.2016.07.003

Wilson, Holly A. y Leticia Gutierrez. 2014. "Does one size fit all? A meta-analysis examining the predictive ability of the Level of Service Inventory (LSI) with Aboriginal offenders». Criminal Justice and Behavior 41(2): 196-219.

Wormith, J. Stephen, Sarah Hogg y Lina Guzzo. 2015. "The predictive validity of the LS/CMI with aboriginal offenders in Canada». Criminal Justice and Behavior 42: 481-508. http://doi.org/10.1177/0093854814552843 